\title{
Asymmetric Atrophy of Paraspinal Muscles in Patients With Chronic Unilateral Lumbar Radiculopathy
}

\author{
Jinmann Chon, MD, PhD, Hee-Sang Kim, MD, PhD, Jong Ha Lee, MD, PhD, Seung Don Yoo, MD, PhD, \\ Dong Hwan Yun, MD, PhD, Dong Hwan Kim, MD, PhD, Seung Ah Lee, MD, PhD, Yoo Jin Han, MD, PhD, \\ Hyun Seok Lee, MD, Young Rok Han, MD, Seonyoung Han, MD, Yong Kim, MD \\ Department of Rehabilitation Medicine, Kyung Hee University Hospital, Seoul, Korea
}

\begin{abstract}
Objective To assess the cross-sectional area (CSA) of the muscles for investigating the occurrence of asymmetry of the paraspinal (multifidus and erector spinae) and psoas muscles and its relation to the chronicity of unilateral lumbar radiculopathy using magnetic resonance imaging (MRI).

Methods This retrospective study was conducted between January 2012 to December 2014. Sixty one patients with unilateral L5 radiculopathy were enrolled: 30 patients had a symptom duration less than 3 months (group A) and 31 patients had a symptom duration of 3 months or more (group B). Axial MRI measured the CSA of the paraspinal and psoas muscles at the middle between the lower margin of the upper vertebra and upper margin of the lower vertebra, and obtained the relative CSA (rCSA) which is the ratio of the CSA of muscles to that of the lower margin of L4 vertebra.

Results There were no differences in the demographics between the two groups. In group B, rCSA of the erector spinae at the L4-5 level, and that of multifidus at the L4-5 and L5-S1 levels, were significantly smaller on the involved side as compared with the uninvolved side. In contrast, no significant muscle asymmetry was observed in group A. The rCSA of the psoas was not affected in either group.

Conclusion The atrophy of the multifidus and erector spinae ipsilateral to the lumbar radiculopathy was observed only in patients suffering from unilateral radiculopathy for 3 months or more.
\end{abstract}

Keywords Paraspinal muscles, Muscular atrophy, Magnetic resonance imaging, Radiculopathy

\footnotetext{
Received November 18, 2016; Accepted February 28, 2017

Corresponding author: Yong Kim

Department of Rehabilitation Medicine, KyungHee University Hospital, 23 Kyungheedae-ro, Dongdaemun-gu, Seoul 02447, Korea. Tel: +82-2-9588565, Fax: +82-2-958-8560, E-mail: okkeun@nate.com

ORCID: Jinmann Chon (http://orcid.org/0000-0002-4186-6623); Hee-Sang Kim (http://orcid.org/0000-0003-4985-0594); Jong Ha Lee (http://orcid. org/0000-0002-7627-6543); Seung Don Yoo (http://orcid.org/0000-0003-4513-2560); Dong Hwan Yun (http://orcid.org/0000-0003-1800-319X); Dong Hwan Kim (http://orcid.org/0000-0002-3812-5509); Seung Ah Lee (http://orcid.org/0000-0002-3426-6259); Yoo Jin Han (http://orcid.org/0000-00033256-9931); Hyun Seok Lee (http://orcid.org/0000-0003-2994-0066); Young Rok Han (http://orcid.org/0000-0002-1680-5802); Seonyoung Han (http:// orcid.org/0000-0002-2218-8150); Yong Kim (http://orcid.org/0000-0003-0950-3774).
}

@ This is an open-access article distributed under the terms of the Creative Commons Attribution Non-Commercial License (http://creativecommons.org/ licenses/by-nc/4.0) which permits unrestricted noncommercial use, distribution, and reproduction in any medium, provided the original work is properly cited. Copyright $\odot 2017$ by Korean Academy of Rehabilitation Medicine 


\section{INTRODUCTION}

Lumbar muscles functionally support and maintain the stability of the spine. The weakness of back muscles is the main cause of recurring low back pain [1].

The psoas muscle is the largest muscle at the cross section of the lower levels of lumbar spine [2]. It stabilize the spine through its potential to generate large compressive forces, thereby increasing the spinal stiffness [3]. Unilateral disc herniation affects the psoas muscle at two locations (the lumbar spine and the hip joint) leading to more pronounced atrophy of the muscle compared with atrophy in other muscles [4]. The multifidus muscle is the most medial part of the lumbar paraspinal muscles [5], unisegmentally innervated by the dorsal ramus medial branch of the segmental nerve [2]. Multifidus muscle atrophy has specifically been studied in relation to disc lesion. Hyun et al. reported a significant decrease in the cross-sectional area (CSA) of the ipsilateral multifidus muscle in patients with unilateral lumbosacral radiculopathy [6]. The erector spinae muscle acts as a global muscle that affects the movement and muscular strength [1]. Since it has a longer lever arm than the multifidus muscle, most of the extensor momentum of the trunk is generated from this muscle rather than the multifidus muscle [7]. Few studies have evaluated the size of the erector spinae muscle in patients with lumbar radicular pain.

Previous studies have shown a significant correlation between the size of a muscle, measured as its CSA, and its ability to generate maximum voluntary isometric force. Since muscular strength is proportional to the CSA of the muscle, the measurement of the CSA of muscle is indirectly used to estimate the strength of the lumbar muscle [1].

To date, there have been several studies of lumbar muscle changes in patients with low back pain or radiculopathy. However, few reports have evaluated the association between the chronicity of unilateral lumbar radiculopathy and the CSA of lumbar muscles, and the studies reporting the association are contradictory. Kim et al. [2] reported that the CSA of multifidus muscle was reduced by disc herniation when symptom duration was three months or more. Conversely, Farshad et al. [8] reported that the asymmetry of multifidus muscle correlated with neither severity nor duration of nerve root compression in lumbar spine. In addition, very few studies have evaluated the changes of both multifidus and erector spinae muscles in patients with unilateral lumbar radiculopathy.

The current study therefore assessed the CSA of paraspinal and psoas muscles using magnetic resonance imaging (MRI), and investigated whether there was an asymmetrical occurrence of the paraspinal (multifidus and erector spinae) and psoas muscles, and whether this feature was related to the chronicity of unilateral lumbar radiculopathy.

\section{MATERIALS AND METHODS}

\section{Subjects}

This was a retrospective study of patients who visited the outpatient clinic of the Department of Rehabilitation Medicine of Kyung Hee University Hospital from January 2012 to December 2014 for symptoms of unilateral lumbar radiculopathy.

Inclusion criteria included: (1) clinical symptoms of unilateral radiculopathy, (2) unilateral L5 radiculopathy seen on electrodiagnostic study of the both lower extremities, and (3) lumbar disc herniation at the L4-5 level on lumbar MRI. The electrodiagnostic criteria for unilateral lumbar radiculopathy included: (1) abnormal spontaneous activity in the unilateral involved paraspinal muscles and/or (2) abnormal spontaneous activity, or abnormal motor unit morphology consistent with nerve injury (polyphasic, large amplitude, increased duration), or reduced recruitment patterns in involved limb muscles innervated by the same myotome but by different nerves.

Exclusion criteria were bilateral symptoms of lower extremities, peripheral polyneuropathy, previous spinal surgery, spinal fracture, spinal cord injury, tumor or infection, and comorbidities influencing physical activities (e.g., severe heart disease, cerebrovascular accident).

Of the total patients screened, 139 were eligible on the basis of their clinical symptoms and electrodiagnostic findings, of which 78 patients were excluded from the study. Finally, 61 subjects ( 31 males and 30 females) were enrolled in this study. The subjects were divided into two groups based on their symptom duration of unilateral radiculopathy: patients with a symptom duration less than 3 months (group A, 30 cases), and patients with a symptom duration of 3 months or more (group B, 31 cases) (Fig. 1). 

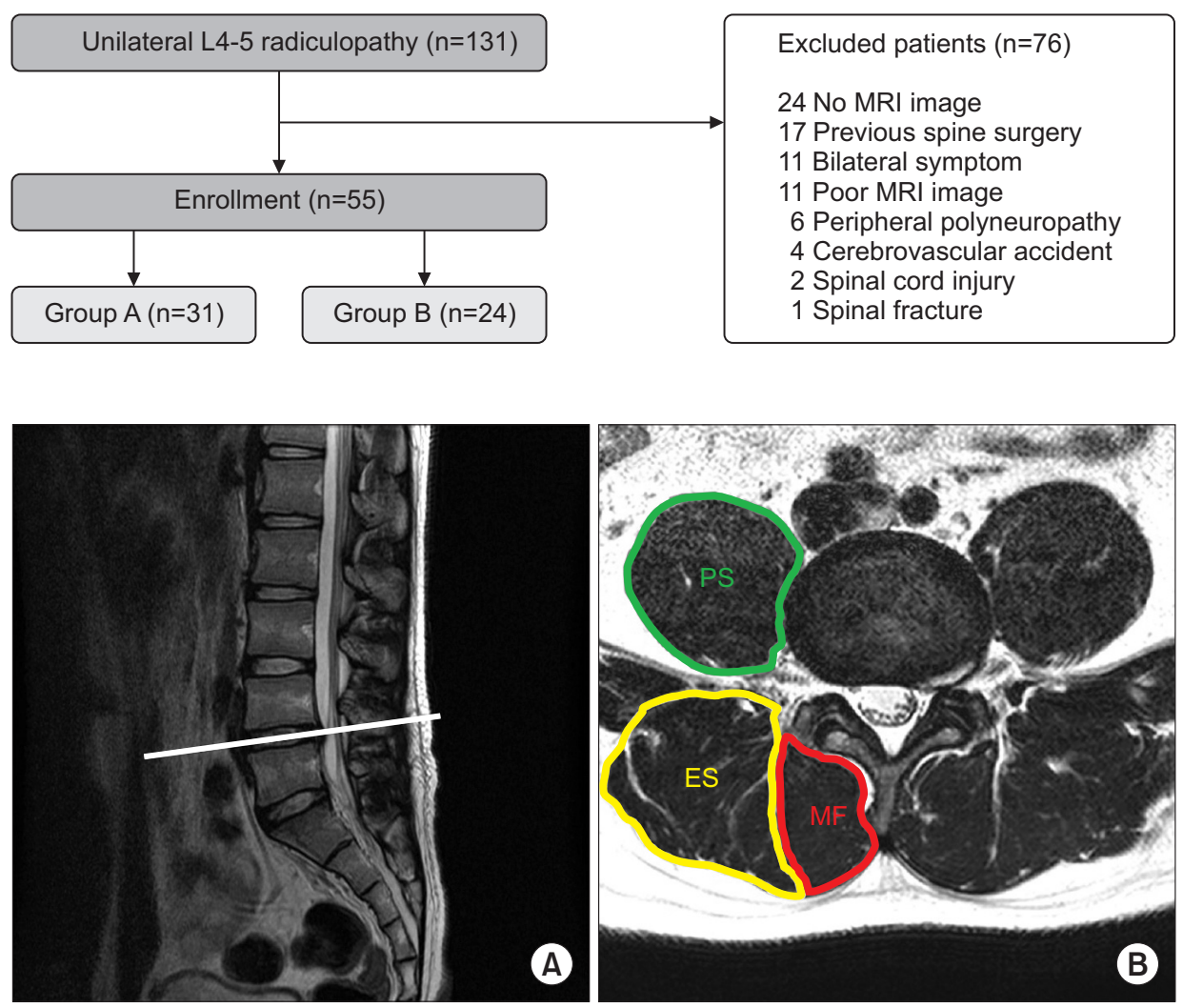

Fig. 1. A flowchart of subjects.

Fig. 2. The cross-sectional area (CSA) of the paraspinal and psoas muscles in a patient with unilateral radiculopathy. (A) Sagittal T2-weighted image shows axial plane at the middle between lower margin of L4 and upper margin of L5 vertebrae. (B) CSA measurement of paraspinal muscles: PS, poas muscle; ES, erector spinae muscle; MF, multifidus muscle.

\section{Measures and procedures}

All patients underwent lumbosacral spine MRI within four weeks before or after the diagnosis of unilateral L5 radiculopathy by electrodiagnostic study. We captured axial T2-weighted images of MRI at the level of the L4-5 and L5-S1 intervertebral disc, which was obtained at the middle between the lower margin of the upper vertebra and upper margin of the lower vertebra. The CSA of both sides of muscles (multifidus, erector spinae, psoas) and lower margin of L4 vertebra were measured by drawing their outlines with the region of interest (ROI) using a PiView program (Infinitt, Seoul, Korea) (Fig. 2). Previous studies had used the relative CSA (rCSA) to compensate for the influence of individual body shape, body weight, and height on the CSA of the muscles [1]. We therefore calculated the rCSA of the muscles, which is defined as the ratio of the CSA of muscles to that of the lower margin of L4 vertebra.

All CSA measurements were performed independently twice by the same person to minimize the potential for error in constructing the polygons around the margins of muscles. The average value of the two measurements was used for statistical analysis. Clinical and electrodiagnos- tic findings of all cases were blinded.

\section{Statistical analysis}

Statistical analysis was performed using the SPSS ver. 13.0 for Windows (SPSS Inc., Chicago, IL, USA). Demographic differences between the two groups were analyzed using chi-square test and independent t-test. Paired t-test was compared the CSA and rCSA between the affected and normal sides in each group. Statistical significance was considered when the $\mathrm{p}$-value was less than 0.05 . All values are presented as mean \pm standard deviation.

\section{RESULTS}

Group A comprised 17 men and 13 women, with mean age 51.0 \pm 13.4 years (range, 25-76 years). Group B comprised 14 men and 17 women, with a mean age $48.2 \pm 15.6$ years (range, 19-71 years). There were no significant differences in demographic characteristics of both groups, except symptom duration (Table 1).

In group A patients with a symptom duration less than 3 months, the differences were statistical not significant 
Table 1. Demographic characteristics of groups A and B

\begin{tabular}{lccc}
\hline & $\begin{array}{c}\text { Group A } \\
(\mathbf{n = 3 0})\end{array}$ & $\begin{array}{c}\text { Group B } \\
(\mathbf{n}=\mathbf{3 1})\end{array}$ & p-value \\
\hline Sex (male:female) & $17: 13$ & $14: 17$ & 0.369 \\
Age $(\mathrm{yr})$ & $51.0 \pm 13.4$ & $48.2 \pm 15.6$ & 0.560 \\
Height $(\mathrm{cm})$ & $165.1 \pm 12.0$ & $164.4 \pm 9.5$ & 0.835 \\
Weight $(\mathrm{kg})$ & $70.0 \pm 15.5$ & $64.2 \pm 14.0$ & 0.229 \\
BMI $\left(\mathrm{kg} / \mathrm{m}^{2}\right)$ & $25.5 \pm 3.8$ & $23.6 \pm 3.8$ & 0.132 \\
Pain $($ VAS, 0-10) & $5.5 \pm 1.7$ & $5.7 \pm 1.5$ & 0.667 \\
Symptom duration (wk) & $5.3 \pm 1.9$ & $33.7 \pm 22.4<0.001^{* * *}$ \\
\hline
\end{tabular}

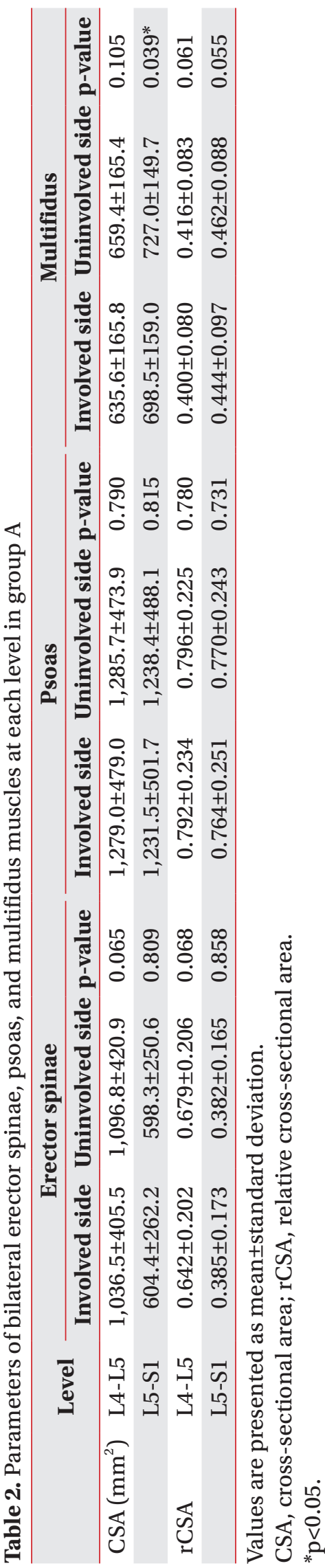

in in any of the muscles, with regards to the rCSA of the involved and the uninvolved sides, at both the L4-5 and L5-S1 levels (Table 2).

In group B patients with a symptom duration of 3 months or more, the rCSA of erector spinae was significantly smaller only at the L4-5 level on the involved side $(0.816 \pm 0.185)$ as compared with the uninvolved side $(0.855 \pm 0.210)(\mathrm{p}<0.05)$, but this difference was not observed at the L5-S1 level.

The rCSA of multifidus muscle at the L4-5 level was significantly smaller on the involved side $(0.463 \pm 0.165)$ as compared with the uninvolved side $(0.500 \pm 0.195)$; a similar significant difference was also observed at the L5S1 level $(0.522 \pm 0.106,0.540 \pm 0.101)(\mathrm{p}<0.05)$.

The rCSA of psoas muscle measured at the L4-5 level was rather small on the involved side $(0.857 \pm 0.252)$ than the uninvolved side $(0.868 \pm 0.215)$, but there were no statistically significant differences in the rCSA of the involved and uninvolved sides at all levels (Table 3).

\section{DISCUSSION}

To date, there have been several studies for multifidus muscle changes in patients with lumbar radiculopathy. However, few reports have evaluated the relationship between the chronicity of unilateral lumbar radiculopathy and the CSA of lumbar muscles, and the results have been contradictory. Farshad et al. [8] reported that the asymmetry of multifidus correlated with neither severity nor duration of nerve root compression in lumbar spine. However, Kim et al. [2] demonstrated that the CSA of the 


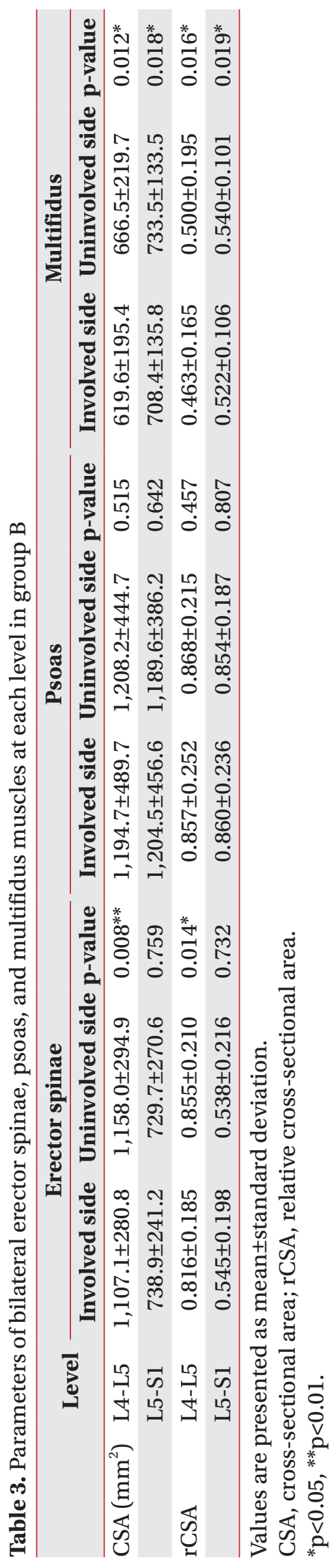

multifidus muscle was reduced by lumbar disc herniation with symptom duration 3 months or more.

In this study, the rCSA of multifidus muscle was influenced by the lumbar unilateral radiculopathy, which was seen only in patients with symptom duration of 3 months or more.

The multifidus muscle is divided into 5 myotomes, each innervated by a single spinal segment. Muscle fibers attaching to the spinous process of a particular vertebra are segmentally innervated by medial branch of the dorsal ramus that originates inferior to the respective vertebra [9]. There have been some studies regarding the extent of multifidus atrophy in patients with lumbosacral radiculopathy. Battie et al. [10] reported that asymmetry of multifidus is most apparent from the muscle measurement at the disc level below the affected disc, and to a lesser degree at the level of herniation because of the unisegmental innervation of multifidus. Similarly, Kang et al. [11] suggested that the most severe atrophy of the multifidus muscle may occur at the mid spinous process level of the vertebra, which is one level below the segmental number of involved nerve root in patients with single level unilateral lumbar radiculopathy.

In this study, multifidus atrophy was shown at the level of L4-5 and L5-S1 intervertebral disc level in unilateral L5 radiculopathy patients with symptom duration of 3 months or more. In our opinion, there are two mechanisms that cause the atrophy of the multifidus muscles: denervation and disuse.

Previous studies revealed that the atrophy of the multifidus muscle was seen at the intervertebral disc level which is one level below the segmental number of involved nerve root, because of the unisegmental innervation of multifidus $[10,11]$. The L5 medial branch of the dorsal ramus innervates the fascicles of multifidus arising from spinous process of L5 vertebra. Therefore, in this study, we suppose that the denervation of the dorsal ramus by L 5 radiculopathy mostly affects the multifidus muscle at the L5-S1 intervertebral disc level.

Furthermore, our study observations revealed that atrophy of the multifidus and erector spinae muscles were at the level of the L4-5 intervertebral disc only, in patients with symptom duration of 3 months or more. At this level, the atrophy of back muscles could mainly be due to disuse. Parkkola et al. [12] suggested that disuse arising with back pain causes disuse atrophy. Other stud- 
ies have established that multifidus atrophy in unilateral back pain is localized rather than generalized, and lumbar dorsal ramus syndrome causes multifidus atrophy in patients with back pain, thus triggering a vicious cycle that promotes atrophy [5]. The maximum CSA of the back muscles are usually observed at the L4-5 intervertebral disc space, and asymmetry of back muscles caused by disuse atrophy are frequently found at this level.

Previous studies reporting the effect of lumbar pathology on psoas size have been contradictory. Dangaria and Naesh [4] reported significant reduction in the CSA of psoas muscle in the affected side only, prominently at the level of disc herniation. Conversely, Danneels et al. [13] reported no change in psoas size in patients with low back pain. In our study, the CSA of the psoas muscle was not influenced by unilateral lumbar radiculopathy. In our opinion, since psoas muscle is not innervated directly by the dorsal ramus of the medial branch, the denervation atrophy may not occur in the psoas muscle. In addition, the psoas muscle acts as a hip flexor which is different from the other two muscles, namely the multifidus and erector spinae. We did not see any atrophy of the psoas muscle, indicating that the muscle atrophy of multifidus and erector spinae was not merely due to general disuse atrophy resulting from global inactivity. The cause of these differences will be examined in the future studies.

A strength of our study was in the diagnosis of lumbosacral radiculopathy. In most previous studies, the patient group with disc herniation and radiculopathy was divided based solely on the clinical symptoms and MRI findings, without electrodiagnostic findings. Only Kang et al. [11] used electrodiagnostic tools in addition to the clinical symptoms and MRI findings. Electrodiagnostic study is an essential method to confirm lumbosacral radiculopathy, and is considered to be a more objective method to reveal the association between muscle atrophy and denervation $[6,14,15]$.

There were several limitations in this study. First, the study sample size was small. Second, because this study was performed retrospectively, standardized assessment of the severity of pain and daily activity were not available. Medical treatment leading to pain relief may also affect the structure of lumbar muscles. This will be examined in the future prospective studies. Finally, only gross measurement techniques were used, and this did not allow for any computation of muscle degeneration due to increased amount of fatty deposit within the muscle.

In conclusion, the study findings suggest that the atrophy of the multifidus and erector spinae muscles ipsilateral to the lumbar radiculopathy are observed only in patients who suffered from unilateral radiculopathy for 3 months or more.

\section{CONFLICT OF INTEREST}

No potential conflict of interest relevant to this article was reported.

\section{REFERENCES}

1. Lee HJ, Lim WH, Park JW, Kwon BS, Ryu KH, Lee JH, et al. The relationship between cross sectional area and strength of back muscles in patients with chronic low back pain. Ann Rehabil Med 2012;36:173-81.

2. Kim WH, Lee SH, Lee DY. Changes in the crosssectional area of multifidus and psoas in unilateral sciatica caused by lumbar disc herniation. J Korean Neurosurg Soc 2011;50:201-4.

3. Barker KL, Shamley DR, Jackson D. Changes in the cross-sectional area of multifidus and psoas in patients with unilateral back pain: the relationship to pain and disability. Spine (Phila Pa 1976) 2004;29: E515-9.

4. Dangaria TR, Naesh O. Changes in cross-sectional area of psoas major muscle in unilateral sciatica caused by disc herniation. Spine (Phila Pa 1976) 1998; 23:928-31.

5. Kader DF, Wardlaw D, Smith FW. Correlation between the MRI changes in the lumbar multifidus muscles and leg pain. Clin Radiol 2000;55:145-9.

6. Hyun JK, Lee JY, Lee SJ, Jeon JY. Asymmetric atrophy of multifidus muscle in patients with unilateral lumbosacral radiculopathy. Spine (Phila Pa 1976) 2007;32: E598-602.

7. Lee HI, Song J, Lee HS, Kang JY, Kim M, Ryu JS. Association between cross-sectional areas of lumbar muscles on magnetic resonance imaging and chronicity of low back pain. Ann Rehabil Med 2011;35:852-9.

8. Farshad M, Gerber C, Farshad-Amacker NA, Dietrich TJ, Laufer-Molnar V, Min K. Asymmetry of the multifidus muscle in lumbar radicular nerve compression. Skeletal Radiol 2014;43:49-53. 
9. Freeman MD, Woodham MA, Woodham AW. The role of the lumbar multifidus in chronic low back pain: a review PM R 2010;2:142-6.

10. Battie MC, Niemelainen R, Gibbons LE, Dhillon S. Is level- and side-specific multifidus asymmetry a marker for lumbar disc pathology? Spine J 2012;12:932-9.

11. Kang JI, Kim SY, Kim JH, Bang H, Lee IS. The location of multifidus atrophy in patients with a single level, unilateral lumbar radiculopathy. Ann Rehabil Med 2013;37:498-504.

12. Parkkola R, Rytokoski U, Kormano M. Magnetic resonance imaging of the discs and trunk muscles in pa- tients with chronic low back pain and healthy control subjects. Spine (Phila Pa 1976) 1993;18:830-6.

13. Danneels LA, Vanderstraeten GG, Cambier DC, Witvrouw EE, De Cuyper HJ. CT imaging of trunk muscles in chronic low back pain patients and healthy control subjects. Eur Spine J 2000;9:266-72.

14. Dillingham TR. Electrodiagnostic approach to patients with suspected radiculopathy. Phys Med Rehabil Clin N Am 2002;13:567-88.

15. Levin KH. Electrodiagnostic approach to the patient with suspected radiculopathy. Neurol Clin 2002;20: 397-421. 